\title{
The Long-Term Clinical Efficacy of Smart Plug in the Treatment of Aqueous Tear Deficiency Dry Eye: Results of a 5-Year Study
}

\author{
Zhaoxia Kuang1,2*, Yong Yao, ${ }^{1,2} 3^{*} \#$, Ye Yuan², Yueting Ma1 ${ }^{1}$ Guanglin Chen ${ }^{1,4}$, \\ Yingying Su${ }^{1}$, Xingkai Zhang1, Vishal Jhanjii, Dennis Lam²
}

\author{
${ }^{1}$ Department of Cornea and Ocular Surface, Joint Shantou International Eye Center of Shantou University and The Chinese \\ University of Hong Kong, Shantou, China \\ ${ }^{2}$ Department of Cornea and Ocular Surface, C-MER Shenzhen Dennis Lam Eye Hospital, Shenzhen, China \\ ${ }^{3}$ C-MER Kunming Eye Hospital, Kunming, China \\ ${ }^{4}$ Department of Cornea and Ocular Surface, Dongguan Aier Eye Hospital, Dongguan, China \\ Email: *jackyao1028@126.com
}

How to cite this paper: Kuang, Z.X., Yao, Y., Yuan, Y., Ma, Y.T., Chen, G.L., Su, Y.Y., Zhang, X.K., Jhanji, V. and Lam, D. (2020) The Long-Term Clinical Efficacy of Smart Plug in the Treatment of Aqueous Tear Deficiency Dry Eye: Results of a 5-Year Study. Open Journal of Ophthalmology, 10, 21-27.

https://doi.org/10.4236/ojoph.2020.101003

Received: November 5, 2019

Accepted: December 27, 2019

Published: December 30, 2019

Copyright $\odot 2020$ by author(s) and Scientific Research Publishing Inc. This work is licensed under the Creative Commons Attribution International License (CC BY 4.0).

http://creativecommons.org/licenses/by/4.0/

\begin{abstract}
Background: Dry eye syndrome, which affects $10 \%$ to $20 \%$ of adults, is a disorder of the tear film and is associated with symptoms of ocular discomfort. Smart Plug is a mechanical treatment in which the tear drainage system is blocked in order to aid in the preservation of natural tears on the ocular surface. Purpose: To evaluate the long-term clinical efficacy of Smart Plug in the treatment of aqueous tear deficiency dry eye. Patients and Methods: Retrospective study. Three hundred and two patients with aqueous tear deficiency dry eye were enrolled and all the patients accepted the punctual plug of Smart plug from Jan. 2011 to Jan. 2016. The clinical symptoms, Schirmer's I test, Fluorescein staining (FL), tear break up time (TBUT), and complications were observed and analyzed. Results: Three hundred and two patients (604 eyes) were treated with Smart Plug (22 cases accepted upper and lower punctual plug, 280 cases only lower punctual plug). There were 109 males (36.1\%) and 193 females (63.9\%) with a mean age of 44.54 years old (Range from 25 to 83 years). Ten cases required the upper Smart Plug after lower punctual plug insertion because of no significantly improved symptoms. Smart plug was removed in 3 patients (1\%) due to tearing. Six patients had mild postoperative tearing and no special treatment was required. Lacrimal canaliculitis occurred postoperatively in 8 cases (2.6\%) (6 lower and 2 upper), and the affected plug was removed and treated with topical antibiotic eye drops. The BUT, SIT and FL scores of all patients were significantly improved from $(2.11 \pm 1.01$, $4.20 \pm 1.07,6.06 \pm 1.97)$ to $(4.34 \pm 1.22,9.01 \pm 1.56,2.33 \pm 1.28)$ respectively
\end{abstract}

${ }^{\star}$ Co-first author. 
after at least 36 months of follow-up (All $P<0.01$ ). The subjective symptoms alleviated after treatment, and the difference was statistically significant $(P<$ 0.05). Conclusion: During a mean follow-up of 4.3 years, Smart plug is an effective method for the treatment of aqueous tear deficiency dry eye in spite of its postoperative complications such as canaliculitis and tearing.

\section{Keywords}

Lacrimal, Dry Eye, Treatment, Smart-Plug, Punctual Plug

\section{Introduction}

Dry eye, which can be divided into two types: aqueous tear deficiency and evaporative dry eye, is the most common ocular surface disease in the world [1] [2]. Aqueous tear deficiency dry eye (ATD) is characterized by unstable tear film caused by the decrease of tear produce of lacrimal glands and the quality of aqueous tears, which can be divided into Sjögren's syndrome and non Sjögren's syndrome [3] [4]. ATD can cause eye symptoms such as irritation, foreign body feeling, fatigue and so on [4]. At present, artificial tears are the most widely used to relieve the symptoms of dry eyes [5]. However, excessive local eye drops are not only inconvenient, but also may damage the tear film and lead to drug ocular toxicity [2] [6]. Punctal occlusion is one of non-pharmacological therapy methods for dry eye syndrome insufficiently treated by other methods. By blocking the lacrimal dots, the naturally secreted tears can be retained in the eyes, thereby alleviating the symptoms of dry eyes [7] [8]. Various materials and plug designs can be used for temporary or semi-permanent packing [9] [10] [11]. A newer kind of a thermodynamic hydrophobic acryl polymer with several features that minimise the risk of complications, Smart Plug (Medennium, Irvine, CA) has been used in clinical in the past few years [10] [12] [13]. Solid at room temperature and softening at body temperature, the Smart Plug can adjust itself to fit the canaliculus, with many advantages which include the lack of plug exposure or extrusion and the ease of removal by lacrimal irrigation. Several publications have demonstrated the efficacy of the SmartPlug in the treatment of dry eye syndrome [10] [11] [12] [13] [14]. However, there are few reports on the long-term effects of its clinical application. In this study, we used Smart Plug to manage ATD, and evaluate its long-term clinical efficacy and safety.

\section{Materials and Methods}

Patients with aqueous tear deficiency dry eye who were admitted to Joint Shantou International Eye Center of Shantou University and The Chinese University of Hong Kong (JSIEC) in the outpatient department from January 2011 to January 2016 were selected. All eligible patients were enrolled after obtaining their written informed consent. The study was approved by the ethics committee of 
JSIEC and all procedures are implemented in accordance with the principles of the Helsinki declaration.

\subsection{Participants}

The subjects of this study were 302 patients (604 eyes) with aqueous tear deficiency dry eye in the outpatient department.

Inclusion criteria: 1) all patients presented more than two kinds of symptoms of conscious discomfort, such as dry eyes, red eyes, photophobia, foreign body sensation, itching, burning sensation, sticky secretion, blurred vision (improvement after blinking), visual fatigue, etc.; 2) examination: tear secretion test (Schirmer I test, SIT) $<10 \mathrm{~mm} / 5 \mathrm{~min} ; 3$ ) tear film break up time (TBUT) $<10 \mathrm{~s}$; 4) corneal fluorescein staining $(\mathrm{FL})$ score $>2$; 5 ) patients require the insertion of lacrimal canaliculus plug whose symptoms cannot be completely relieved using artificial tears more than 3 times daily [14].

Exclusion criteria:

All patients were excluded from lacrimal duct disease, active ocular surface inflammation, refractive corneal surgery and other obvious eye diseases.

\subsection{Methods}

1) The lacrimal duct was rinsed preoperatively to make sure the lacrimal duct was unobstructed. Topical anesthesia was performed 2 to 3 times with proparacaine hydrochloride eye drops (s.a. ALCON-COUVREUR n.v). Expose tear spots and expand them if necessary. The Smart Plug was vertically inserted into the lacrimal puncture using microscopic tweezers. When about $2 / 3$ of it enter lacrimal canaliculi, the Smart Plug was shortened and distended and completely retracted into the lacrimal tubule under the action of body temperature.

2) All patients included in this study were followed up 7 days, 1 month, 3 months, 6 months, 12 months and 36 months after surgery. The results of the last review are used to count the results. Routine inspections include: a) conjunctival congestion, photophobia, dryness, burning, foreign body sensation, fatigue, epiphora and other conditions were recorded; b) Schirmer's I Test (SIT): Bend the filter paper along the upper end mark and place it on the middle and outer $1 / 3$ of the lower eyelid. Ask patients to close their eyes for $5 \mathrm{~min}$, then take out the filter paper and record the wetting length of filter paper. c) BUT: One drop of fluorescein sodium was placed in the conjunctival sac and the patient was instructed to blink several times. The time from eye opening after the last blink to the first dry spot on the surface of the tear film stained with fluorescein was recorded as the measured value of BUT. Check three times and take the average result. d) FL standard for evaluation: Cornea is divided into 4 quadrants, each quadrant $0-3$ points, a total of $0-12$ points.

Postoperative management

Topical $0.1 \%$ sodium hyaluronate eye drops (Santen Pharmaceutical Co., Ltd.) was used 3 - 4 times a day postoperatively. If there is a lacrimal canaliculitis, re- 
move the punctual plug and topical use of $0.5 \%$ Levofloxacin eye drops (Santen Pharmaceutical Co., Ltd. Noto Plant) 4 times daily.

\subsection{Statistical Analysis}

SPSS 16.0 statistical software (version 2.12.12) was used for statistical analysis. The paired $\mathrm{T}$ test was used to compare the difference of patients before and after treatment, where $p<0.05$ was considered to be statistically significant.

\section{Results}

Three hundred and two patients (604 eyes) were treated with Smart Plug (22 cases accepted upper and lower punctual plug, 280 cases only lower punctual plug). There were 109 cases (218 eyes) in male and 193 cases (386 eyes) in female. The mean age is $44.54 \pm 13.86$ years old (from 25 to 83 years). Upper and lower lacrimal duct embolization was performed simultaneously in 12 severe cases and obtain satisfactory result. Ten cases required the upper Smart Plug after lower punctual plug because of no significantly improved symptoms. The symptoms of discomfort were significantly reduced after the reimplantation of upper punctual plug in all eyes 3 month later. Nine patients complained of tears after surgery. Smart plug were removed in 3 patients because of tearing, and no special treatment was required in the remain six patients. Lacrimal canaliculitis occurred postoperatively in 8 cases (6 lower and 2 upper) and the affected plug was removed and treated with topical antibiotic eye drops. Culture positive specimens were 7 of 8 (87.5\%), with 4 of Streptococcus species (57.1\%), 2 of Staphylococcus species (28.5\%), and 1 of Actinomyces (14.3\%).

In the median of 4.3 years ( $3-8$ years) follow-up period, the difference was statistically significant in the scores of BUT, SIT and FL compared with preoperative $(P<0.01)$ and the subjective symptoms of patients were reduced (Table 1$)$.

\section{Discussion}

In addition to medication, blocking the drainage system of the lacrimal duct is the most common treatment for dry eye. It helps preserve any remaining natural tears, which by far have the best moisture and nutrient capacity on the surface of the eye. Our study showed that in the long-term Smart plug lacrimal canaliculi had a definite effect in the treatment of ATD with fewer complications.

At present, the main treatment methods for dry eyes include artificial tears, immunosuppressive agents, lacrimal duct embolization, and submandibular

Table 1. Comparison before and after treatment in patients with BUT, SIT and FL scores.

\begin{tabular}{cccc}
\hline & Before Treatment & after treatment & p \\
\hline BUT & $2.11 \pm 1.01$ & $4.34 \pm 1.22$ & $<0.01$ \\
SIT & $4.20 \pm 1.07$ & $9.01 \pm 1.56$ & $<0.01$ \\
FL scores & $6.06 \pm 1.97$ & $2.33 \pm 1.28$ & $<0.01$ \\
\hline
\end{tabular}

${ }^{*}$ The last time follow-up. 
gland transplantation, and so on [6] [15]. Artificial tears are mainly targeted at patients with mild to moderate dry eyes [16]. Although it can temporarily relieve the symptoms of patients with dry eyes, it still has many shortcomings: 1) Compliance gradually declined in many patients because of the long and frequent administration; 2) Most of artificial tears contain preservatives, and frequent using over a long period of time can aggravate ocular surface damage; 3) Artificial tears do not have the antibacterial components in normal tears, such as the lysozyme, immunoglobulin and epidermal growth factor. These compounds can inhibit or kill pathogenic microorganisms, exert immune function, and initiate the normal growth and differentiation of ocular epithelium [17]; 4) Long-term frequent use of artificial tears will wash away the normal tear film, thus speeding up the evaporation of tears [18].

In recent years, more and more studies have been reported that the SmartPlug lacrimal tubule has achieved good efficacy in the treatment of ATD dry eyes [12] [14] [19]. The tear loss can be slowed down and the duration of the tear in the conjunctival sac can be prolonged by blocking the canaliculi and blocking the tear flow [7] [8] [11] [20]. Human tears contain growth factors, immunoglobulin, and other ionic components that improve eye defense. The results of this study show, the scores of BUT, SIT and FL have been improved significantly compared with that of preoperative after at least 36 months follow up in the treatment of ATD with Smart plug. Satisfactory results were obtained because the lower tear river was significantly widened and patient's subjective discomfort symptoms improved or disappeared.

It was optional to conduct single lower, or combined upper and lower Smart Plug lacrimal canaliculi embolization according to the severity of the ATD. It is better to combine with the upper and lower smart-plug, and the effect of simple lower canaliculi plug is limited, especially in severe patients with SjÖgren's syndrome. In our stduy, 10 patients performed the second Smart Plug because of no obvious symptomatic improvement, and the symptoms score of discomfort were significantly reduced in all eyes 3 month later.

Lacrimal canaliculitis is usually a challenge in the long-term for both patients and clinicians [13]. Hill et al. [21] reported a $4.73 \%$ prevalence of canaliculitis per SmartPlug within an average follow-up time of 3 years. Klein-Theyer et al. [13] reported a higher prevalence of $16.6 \%$ per SmartPlug insertion occurred after a median of 4.7 years follow-up. They concluded that only long-term follow-up periods, such as those up to $10.5 \mathrm{y}$ as used in their study, can identify the majority of complications associated with smart plugs. In this study Lacrimal canaliculitis occurred postoperatively in 8 cases $(2.6 \%)$ during a mean follow-up of 4.3 years. Most of them can be cured after the removal of lacrimal canaliculus emboli and local application of antibiotics. No canaliculotomy was necessary to cure the condition. In addition, no lacrimal duct granuloma was found in the at least 36 months follow-up.

Our study was a retrospective, non-controlled clinical study, with a small 
number of cases collected and different follow-up times. In the future, we need to expand the sample size and conduct multi-center, prospective and controlled clinical studies to obtain more scientific results.

\section{Conclusion}

In conclusion, this study shows that the Smart Plug lacrimal tubule is one of the most effective long-term methods in the treatment of ATD. Appropriate lacrimal duct embolization can be selected according to the severity of the disease. Considering the sale sample size of this study, a larger sample and multi-center clinical studies will be required to evaluate the comprehensive and accurate clinical efficacy in the future.

\section{Fund Support}

This study was supported by Grants from Guangdong science and technology research fund (A2017486).

\section{Conflicts of Interest}

The authors of this manuscript have no conflicts of interest to disclose with the products mentioned.

\section{References}

[1] Craig, J.P., Nichols, K.K., Nichols, J.J., et al. (2017) TFOS DEWS II Definition and Classification Report. The Ocular Surface, 15, 276-283. https://doi.org/10.1016/j.jtos.2017.05.008

[2] Dogru, M., Nakamura, M., Shimazaki, J., et al. (2013) Changing Trends in the Treatment of Dry-Eye Disease. Expert Opinion on Investigational Drugs, 22, 1581-1601. https://doi.org/10.1517/13543784.2013.838557

[3] Shimazaki, J. (2018) Definition and Diagnostic Criteria of Dry Eye Disease: Historical Overview and Future Directions. Investigative Ophthalmology \& Visual Science, 59, Des7-des12. https://doi.org/10.1167/iovs.17-23475

[4] Lemp, M.A., Baudouin, C., Baum, J., et al. (2007) The Definition and Classification of Dry Eye Disease: Report of the Definition and Classification Subcommittee of the International Dry Eye Workshop (2007). The Ocular Surface, 5, 75-92. https://doi.org/10.1016/S1542-0124(12)70081-2

[5] Moshirfar, M., Pierson, K., Hanamaikai, K., et al. (2014) Artificial Tears Potpourri: A Literature Review. Clinical Ophthalmology (Auckland, N.Z.), 8, 1419-1433. https://doi.org/10.2147/OPTH.S65263

[6] Marshall, L.L. and Roach, J.M. (2016) Treatment of Dry Eye Disease. The Consultant Pharmacist. The Journal of the American Society of Consultant Pharmacists, 31, 96-106. https://doi.org/10.4140/TCP.n.2016.96

[7] Ervin, A.M., Law, A., Pucker, A.D., et al. (2017) Punctal Occlusion for Dry Eye Syndrome. Cochrane Database of Systematic Reviews, No. 9, CD006775. https://doi.org/10.1002/14651858.CD006775.pub3

[8] Ervin, A.M., Law, A., Pucker, A.D., et al. (2019) Punctal Occlusion for Dry Eye Syndrome: Summary of a Cochrane Systematic Review. The British Journal of Ophthalmology, 103, 301-306. https://doi.org/10.1136/bjophthalmol-2018-313267 
[9] Tai, M.C., Cosar, B.C., Cohen, E.J., et al. (2002) The Clinical Efficacy of Silicone Punctal Plug Therapy. Cornea, 21, 135-139. https://doi.org/10.1097/00003226-200203000-00001

[10] Said, A.M.A., Farag, M.E., Abdulla, T.M., et al. (2016) Corneal Sensitivity, Ocular Surface Health and Tear Film Stability after Punctal Plug Therapy of Aqueous Deficient Dry Eye. International Journal of Ophthalmology, 9, 1598-1607.

[11] Tong, L., Beuerman, R., Simonyi, S., et al. (2016) Effects of Punctal Occlusion on Clinical Signs and Symptoms and on Tear Cytokine Levels in Patients with Dry Eye. The Ocular Surface, 14, 233-241. https://doi.org/10.1016/j.jtos.2015.12.004

[12] Chen, S.X. and Lee, G.A. (2007) SmartPlug in the Management of Severe Dry Eye Syndrome. Cornea, 26, 534-538. https://doi.org/10.1097/ICO.0b013e318038d2cd

[13] Klein-Theyer, A., Boldin, I., Rabensteiner, D.F., et al. (2015) Prevalence of Canaliculitis after Smartplug Insertion during Long-Term Follow-Up. The British Journal of Ophthalmology, 99, 1134-1136.

https://doi.org/10.1136/bjophthalmol-2014-306290

[14] Li, G., Wu, X.Y., Fang, Y., et al. (2010) Application of Smart Plug Lacrimal Plug in the Treatment of Dry Eye. Journal of Southern Medical University, 30, 2370-2372.

[15] Brown, S.M. (2007) Cure for Dry Eye. Ophthalmology, 114, 1585-1586. https://doi.org/10.1016/j.ophtha.2007.04.019

[16] Asbell, P.A. (2006) Increasing Importance of Dry Eye Syndrome and the Ideal Artificial Tear: Consensus Views from a Roundtable Discussion. Current Medical Research and Opinion, 22, 2149-2157. https://doi.org/10.1185/030079906X132640

[17] Tong, L., Petznick, A., Lee, S., et al. (2012) Choice of Artificial Tear Formulation for Patients with Dry Eye: Where Do We Start? Cornea, 31, S32-S36. https://doi.org/10.1097/ICO.0b013e318269cb99

[18] Pucker, A.D., Ng, S.M. and Nichols, J.J. (2016) Over the Counter (OTC) Artificial Tear Drops for Dry Eye Syndrome. Cochrane Database of Systematic Reviews, 2, CD009729. https://doi.org/10.1002/14651858.CD009729.pub2

[19] Kojima, T., Matsumoto, Y., Ibrahim, O.M.A., et al. (2014). Evaluation of a Thermosensitive Atelocollagen Punctal Plug Treatment for Dry Eye Disease. American Journal of Ophthalmology, 157, 311-317.e1. https://doi.org/10.1016/j.ajo.2013.10.019

[20] Shi, S., Chen, W., Zhang, X., et al. (2013) Effects of Silicone Punctal Plugs for Tear Deficiency Dry Eye Patients. Chinese Journal of Ophthalmology, 49, 151-154.

[21] Hill III, R.H., Norton, S.W. and Bersani, T.A. (2009) Prevalence of Canaliculitis Requiring Removal of SmartPlugs. Ophthalmic Plastic \& Reconstructive Surgery, 25, 437-439. https://doi.org/10.1097/IOP.0b013e3181b80d63 